\title{
TTR
}

Traduction, terminologie, re?daction

\section{Conclusions : " La Tâche du traducteur " de Walter Benjamin}

\section{Paul de Man}

Volume 4, numéro 2, 2e semestre 1991

Traduire la théorie

URI : https://id.erudit.org/iderudit/037092ar

DOI : https://doi.org/10.7202/037092ar

Aller au sommaire du numéro

Éditeur(s)

Association canadienne de traductologie

ISSN

0835-8443 (imprimé)

1708-2188 (numérique)

Découvrir la revue

Citer cet article

de Man, P. (1991). Conclusions : « La Tâche du traducteur » de Walter

Benjamin. TTR, 4(2), 21-52. https://doi.org/10.7202/037092ar d'utilisation que vous pouvez consulter en ligne.

https://apropos.erudit.org/fr/usagers/politique-dutilisation/ 


\section{Conclusions: «La Tâche du traducteur» de Walter Benjamin}

(Messenger Lecture, Université Cornell, 4 mars 1983)

\section{Paul de Man}

\section{Note du traducteur}

La tâche du traducteur, ici, était multiple. Il s'agissait d'abord de respecter la dimension orale du texte que le responsable de la transcription lui-même avait conservée. Il fallait en outre laisser à la langue de Paul de Man la singularité due au fait qu'il s'exprimait en anglais, tout en retrouvant, le cas échéant, les tournures françaises qui se laissaient entrevoir. Par ailleurs, le vocabulaire employé étant largement philosophique, il était important d'utiliser le lexique habituel attaché aux auteurs ou aux courants traités: Kant, Hegel, Heidegger ou la phénoménologie. Enfin, le texte s'appuyant sur plusieurs langues (anglais, allemand et français), nous devions marquer à notre tour ces présences linguistiques tout en variant leurs occurrences par rapport au texte original puisque la langue d'arrivée était le français. Nous avons été amené à ajouter des parenthèses et à jouer d'une ponctuation qui, si elle n'est pas celle du texte anglais, échappe aussi parfois aux usages habituels du français. Nous avons cru bon de traduire également la note qui précédait l'édition du texte en anglais. Les chiffres entre parenthèses suivant les citations de Benjamin renvoient au texte original ou aux traductions anglaise et française de $\mathrm{H}$. Zohn et $\mathrm{M}$. de Gandillac. Nous avons substitué là traduction de $M$. de Gandillac à celle de $\mathrm{H}$. Zohn

1. Panu en anglais dans Yale French Studies (vol. 69, 1985, pp. 25-46). 
citée dans le texte original quand la précision du développement de Paul de Man n'en souffrait pas.

A. $\mathbf{N}$.

\section{Avant-propos à l'édition originale}

Ce texte est une transcription annotée de la dernière conférence des six Messenger Lectures données à l'Université Cornell en février et mars 1983. Il est rédigé à partir de trois enregistrements auxquels s'ajoutent huit pages de notes manuscrites. Outre des différences dans les détails, la formulation et l'accentuation rhétorique, les notes ne s'éloignent sensiblement des enregistrements que dans la dernière feuille où de Man écrit: «Im Angang war das Wort und das Wort war bei Gott/ Dasselbe war bei Gott/ ohne Daselbe» (les deux demiers mots étant soulignés) le début de la traduction de Luther de l'évangile de Saint-Jean, cité par Benjamin en grec et auquel de Man fera référence dans la période de questions ayant suivi la conférence. Le présent texte garde des traces du cadre dans lequel fut donnée la conférence, notamment en ce qui concerne les références aux traductions précédentes.

Bien que la tâche du transcripteur - donner à un texte non-écrit une après-vie qui le rende canonique - ne puisse être entreprise qu'en surseoyant à l'idéal de fidélité qui devrait la guider, j'ai tenté autant que possible de résister à la nécessité de figer les passages qui apparaissaient encore en voie de formulation. L'anglais parfois non-conforme de de Man a été conservé à quelques modifications près introduites par souci de cohérence. Quelques phrases et paragraphes ont dû être réagencés. Les solécismes et les répétitions ont cependant été conservés, même s'ils peuvent incommoder la lecture, quand le texte écrit tendait par trop à s'éloigner de l'oralité. J'ai ainsi tenté de faire passer au lecteur une partie des difficultés et des risques de la reconstruction. Les omissions et les corrections répondent à ce principe. La ponctuation introduite est moins dépendante de l'usage que du souci đe fidélité inhérent à l'acte de transcription. Il s'agissait pour moi de reproduire le rythme de l'oralité et d'offrir le plus de lectures possibles, les ambiguités devant plus à la nature instable du procédé qu'au désir d'être fidèle à la pensée de de Man. Le découpage des paragraphes suit en général les pauses adoptées oralement ainsi que les répétitions des propositions principielles qui semblaient pour de Man marquer les 
développements de l'argumentation. Ces coupures se retrouvent partiellement dans les notes manuscrites. A l'exception de quelques passages tirés par de Man de la traduction de Zohn, les citations dans ce texte reproduisent ses traductions improvisées, parfois éloignées des traductions anglaises citées dans mes notes. Cet article est publié avec l'aimable autorisation des Presses de l'Université du Minnesota auxquelles il avait été promis.

William D. Jewett

J'avais d'abord pensé laisser ouverte cette dernière session pour tirer des conclusions et accueillir la discussion. Je garde l'espoir d'une discussion mais j'ai abandonné l'idée de pouvoir tirer des conclusions. Plutôt que d'essayer de conclure (ce qui est toujours terriblement décevant), il me semble préférable de répéter une fois de plus ce que j'ai dit depuis le début, en utilisant un autre texte afin d'avoir une nouvelle version, une nouvelle formulation de certaines des questions qui nous ont occupés tout au long de cette série de conférences. Il me semblait que le texte en question, "la Tâche du traducteur» de Benjamin, était un texte très connu, c'est-à-dire qu'il connaissait une très large diffusion mais aussi que dans la profession vous ne pouviez vous faire un nom à moins d'avoir dit quelque chose sur ce texte. Puisque la plupart d'entre nous ont sans doute tenté de dire quelque chose à son sujet, laissez-moi essayer à mon tour et puisqu'il est probable que certains d'entre vous soient bien plus avancés que moi, $j$ 'attends vos questions ou vos suggestions. Aussi, loin de conclure ou de formuler des propositions très générales, je voudrais rester assez près de ce texte-là et voir ce qu'il peut en sortir. Puisque je dis rester près de ce texte, dans la mesure où il s'agit d'un texte sur la traduction, je vais avoir besoin (c'est pourquoi j'ai tous ces livres) de traductions de ce texte. Car si vous avez un texte disant qu'il est impossible de traduire, il est très intéressant de voir ce qui arrive quand on traduit ce texte. Et ces traductions confirment, avec brio, au-delà de tout ce que je pouvais espérer, qu'il est impossible de traduire, comme vous allez le voir dans un instant. 
Quoi qu'il en soit, j'ai cependant placé tout cela à l'intérieur d'une sorte de cadre, de cadre historique. Puisque les questions concernant l'histoire ont fréquemment surgi, j'ai pensé qu'il serait bon de nous placer dans un cadre historique ou pseudo-historique et puis de partir de là. Je commencerai donc par un problème récurrent en histoire et dans l'historiographie, le problème de la modernité. J'utilise en guise d'introduction un petit essai du philosophe allemand Gadamer qui, dans un recueil intitulé Aspekte der Modernität, a publié, il y a de nombreuses années, d'intéressants articles sous le titre de «Die philosophischen Grundlagen des zwanzigsten Jahrhunderts» [les Fondements philosophiques du vingtième siècle]. Gadamer y pose la question, peut-être naive mais certainement pertinente, de savoir si ce qui est fait en philosophie au vingtième siècle differe substantiellement de ce qui a été fait auparavant et si donc il est sensé de parler d'une modernité de la spéculation philosophique au vingtième siècle. Il estime que le thème général, la visée générale de la philosophie contemporaine consiste en une critique du concept de sujet. On ne dirait peut-être pas la même chose aujourd'hui, ce qui fait que l'essai peut sembler dater un peu, mais le propos est toujours pertinent. Il se demande donc si la manière dont la critique du concept est traitée de nos jours en philosophie differe substantiellement de la manière dont l'abordaient les prédécesseurs de la philosophie contemporaine, les tenants de la philosophie idéaliste allemande - certains des auteurs dont nous nous sommes préoccupés, tels que Kant, Hegel et d'autres encore. Il écrit ceci qui sera notre point de départ:

La critique du concept de sujet qui est tentée en notre siècle est-elle quelque chose d'autre, de différent d'une simple répétition de ce qui avait été accompli par l'idéalisme allemand - et, disons-le, avec, dans notre cas, beaucoup moins de puissance d'abstraction et sans la force conceptuelle qui caractérisait le mouvement antérieur? [notre traduction] ${ }^{2}$

2. Le texte allemand qui parut dans Aspekte der Modernität (Gottingen, Vanderhoeck \& Ruprecht, 1965, pp. 77-100) est repris dans les Kleine Schriften (Tübingen, J.C.B. Mohr, 1967, v. 1, pp. 131-148). On en trouve une traduction anglaise dans le recueil Philosophical Hermeneutics (tr. D.E. Linge, Berkeley, U. of California Press, 1976, pp. 107-129). Cf. Kleine Schriften, v. 1, p. 141; Philosophical 
Ne faisons-nous que répéter? Et il répond, ce qui est étonnant: «Ce n'est pas le cas.» Ce que nous faisons est réellement quelque chose de nouveau, quelque chose de différent et nous pouvons nous poser en tant que philosophes modernes. Il dégage trois domaines dans lesquels nous - les philosophes contemporains - et lui, Gadamer, sommes en avance sur nos prédécesseurs et il caractérise cette triple avance comme une décroissance en termes de naïveté. Il nous semble désormais, si nous retournons à Hegel ou Kant, y trouver une certaine naïveté que nous avons dépassée. Il distingue entre trois types de naïveté qu'il nomme Naivität des Setzens (naivveté du postulat), Naivität der Reflexion (naiveté de la réflexion) et Naivität des Begriffs (naïveté du concept).

Très brièvement, disons que ce qui est entendu par le premier aspect, une certaine naïveté de postulation, consiste en une critique que nous avons été en mesure de développer concernant la perception pure et le discours purement affirmatif, en rapport avec la question du sujet. Nous sommes maintenant en avance sur Hegel en ce que nous savons mieux désormais que le sujet ne maîtrise pas ses propres énonciations; nous sommes davantage conscients qu'il est naif de supposer que le sujet est réellement en contrôle de son propre discours; nous savons que ce n'est pas le cas. Il apporte cependant une légère réserve à ce propos: la compréhension nous est néanmoins, jusqu'à un certain point, possible grâce à un processus herméneutique par lequel la compréhension, au travers d'un processus historique, peut corriger les présupposés qu'elle accueillait sur elle-même. Gadamer, disciple de Heidegger, nous offre là un développement de la notion de cercle herméneutique, dans lequel le sujet est aveugle à sa propre énonciation mais où le lecteur qui est conscient de l'historicité de cette cécité peut néanmoins retrouver la signification, peut retrouver un certain degré de contrôle sur le texte au moyen de cette structure herméneutique particulière. Ce modèle de compréhension est plus avancé que le modèle hégelien exactement de la même manière qu'on pourrait dire que l'herméneutique de Heidegger est plus avancée que l'herméneutique de Hegel, au sens de Gadamer.

Hermeneutics, p. 119. Il en existe une traduction française parue dans le recueil la Sécularisation de la pensée, sous la direction de G. Vattimo (Paris, Le Seuil, 1988, pp. 197-217). 
Il parle ensuite de la naïveté de réflexion et pousse plus loin ce qu'il avait développé précédemment, à savoir qu'il pose la possibilité maintenant d'une historicité de la compréhension, d'une manière qui n'est pas offerte à l'auto-réflexion individuelle. Hegel, en un sens, n'était pas assez historique; chez lui c'est encore trop le sujet lui-même qui est à l'origine de sa propre compréhension alors que l'on est conscient aujourd'hui de la complexité des rapports entre le sujet et son discours. Si, dans sa première analyse, il renvoyait à l'apport de Hegel, il renvoie surtout ici à ses propres travaux: historiciser la notion de compréhension en considérant la compréhension (ainsi que le développera ultérieurement la Rezeptionsästhetik dont Gadamer est en grande partie l'inspirateur) comme un processus entre l'auteur et le lecteur par lequel le lecteur acquiert une compréhension du texte en prenant conscience de l'historicité du mouvement établi entre le texte et lui-même. Sur ce point également Gadamer affirme que quelque chose de nouveau s'est mis en place de nos jours. Il est vrai que l'accent porté sur la réception, l'accent porté sur la lecture sont caractéristiques de la théorie contemporaine et peuvent prétendre à la nouveauté.

Il parle enfin de la naïveté du concept: là c'est la question du rapport entre le discours philosophique et les données thétoriques ou autres, appartenant davantage au domaine du discours ordinaire ou du langage commun, qui n'a pas été, avec Kant et Hegel, examinée de manière critique. Nous en avons fait hier allusion à un exemple avec le problème de l'hypotyposis soulevé par Kant qui nous invite à devenir conscients de l'usage des métaphores dans notre propre discours philosophique. Ce genre de question, au moins mentionne par Kant mais beaucoup moins par Hegel, est maintenant plus ou moins développé. Gadamer fait allusion à Wittgenstein et aussi indirectement à Nietzsche. Nous ne pensons plus, dit-il, que les langages conceptuel et ordinaire sont distincts; nous avons maintenant une conception de la problématique du langage qui est moins naïve en ce qu'elle reconnaît le degré de dépendance du langage philosophique par rapport au langage ordinaire et leur proximité. Voici donc la modernité qu'il présente et qu'il développe selon ces trois directions.

Or bien que ce modèle soit en partie kantien par sa perspective critique, il est encore très hégélien. Le schème ou le concept de 
modernité se présente comme dépassement d'une certaine absence de conscience, d'une certaine naïveté, au moyen d'une négation critique, au moyen d'une analyse critique entraînant la négation de certains rapports positifs et la réalisation d'une nouvelle conscience. Cette présentation revient à établir un nouveau discours qui prétend dépasser ou renouveler une certaine problématique. Ce modèle est très traditionnellement hégélien dans le sens où le développement de la conscience est toujours présenté comme une sorte de dépassement d'une certaine naïveté et l'accession de la conscience à un autre niveau. Il est traditionnellement hégélien, ce qui ne veut pas dire qu'il se trouve chez Hegel mais que c'est dans Hegel ce qu'on enseigne de Hegel. De fait, Gadamer conclut son essai par une référence à Hegel:

Le concept d'esprit, que Hegel emprunte à la tradition spirituelle chrétienne, est encore le fondement de la critique du sujet et de l'esprit subjectif qui apparaît l'objectif principal de la période post-hégélienne, c'est-à-dire moderne. Ce concept d'esprit (Geist), qui transcende la subjectivité de l'ego, trouve sa véritable place dans le phénomène du langage qui s'affirme de plus en plus comme le centre de la philosophie contemporaine. [notre traduction] ${ }^{3}$

La philosophie contemporaine se préoccupe d'aller au-delà de Hegel dans les termes de Hegel, en se basant sur la démarche $e^{4}$ hégélienne, la dialectique hégélienne telle qu'elle s'attache plus spécifiquement à la question du langage. C'est ainsi que la modernité est ici définie, comme un hégélianisme plus axé sur les dimensions linguistiques.

Si nous comparons le concept de modernité avancé ici par Gadamer, concept critique, dialectique, non-essentialiste (puisque pragmatique dans une certaine mesure: il y est tenu compte du langage commun), avec le texte de Benjamin sur le langage, «la Tâche du traducteur», Benjamin apparaîtra à première vue comme fortement rétrograde. Il apparaitra messianique, prophétique, religieusement messianique, d'une manière qui pourrait bien sembler un retour à la

3. Cf. Kleine Schriften, v.1, p. 148; Philosophical Hermeneutics, p. 128.

4. En français dans le texte. 
naïveté dénoncée par Gadamer. Il a de fait été critiqué pour cela. Ce retour remonterait en fait à un stade bien plus ancien que celui de Kant, Hegel et l'idéalisme. La première impression que vous recevez du texte de Benjamin est qu'il s'agit d'une déclaration messianique, prophétique, qui serait très éloignée de cet esprit froid et critique qui, de Hegel à Gadamer, est tenu pour l'esprit de la modernité. De fait, en lisant le texte, vous êtes frappé par le ton messianique, par la figure du poète comme une figure presque sacrée, une figure accueillant un langage sacré. Toutes les références, dans le texte, à des poètes précis soulignent avec insistance ce trait. Ceux qui sont mentionnés sont des poètes associés à une fonction sacerdotale, presque ecclésiastique, spirituelle de la poésie: c'est le cas de Hőlderlin, de George et de Mallarmé, tous trois très présents dans cet essai.

(Puisque je le mentionne, la présence de George est très sensible dans le texte - un nom qui a perdu de nos jours beaucoup de son importance mais qui à l'époque en Allemagne était encore considéré comme le poète le plus important, la figure la plus centrale, bien qu'en 1923 ou i924, quand ceci fut écrit, le phénomène allait déjà vers sa fin. Par exemple, Benjamin cite Pannwitz, un disciple de George, à la fin du texte. Et sa référence à George est pertinente: avec George, ce qui était revendiqué, c'était le poète comme une sorte de prophète, une sorte de figure messianique. George se prend très au sérieux à cet égard, il se voit pour le moins comme un mélange de Virgile et de Dante, et encore plus s'il le faut; il a donc une conception très haute du rôle du poète et de lui-même, soit dit en passant - et de tous les avantages qui en découlent. Mais cet état d'esprit imprègne tout le discours académique allemand et une certaine conception de la poésie qui étaient de rigueur à l'époque. On en trouve beaucoup l'influence sur la façon dont Benjamin approche le problème, du moins dans une lecture superficielle. Il en est de même des références à Hölderlin qui à l'époque était une découverte de George et de son groupe; ces mentions révèlent une certaine conception messianique, spirituelle de Hölderlin. On en trouvera encore de nombreuses traces chez Heidegger qui, après tout, dédia ses commentaires sur Hőlderlin à Norbert von Hellingrath qui était un disciple de George et un membre de son cercle et qui fut, comme vous le savez, le premier à éditer Hölderlin. J'ai esquissé sommairement ce petit tableau - qui vous est peut-être familier, totalement redondant - afin de montrer que le climat, l'atmosphère 
dans laquelle cet essai fut écrit cède volontiers et fréquemment à la notion du poétique comme sacré, du langage comme sacré, de la figure du poète comme, dans une certaine mesure, une figure sacrée.)

Cette influence n'est pas simplement présente sous forme de traces chez Benjamin, elle semble presque faire partie du message énoncé. Cette notion de la poésie comme langage sacré, ineffable prend peut-être sa forne extrême dès le début, dans la manière catégorique dont Benjamin rejette toute notion de la poésie comme orientée, de quelque façon, vers un public ou un lecteur. Ce passage a soulevé la colère des tenants de la Rezeptionsästhetik qui analysent le problème de l'interprétation poétique du point de vue du lecteur. Stanley Fish ou M. Riffaterre, dans ce pays, suivent cette ligne dans une certaine mesure mais ce sont bien sûr Jauss et ses disciples qui vont le plus loin en ce sens. À leurs yeux une phrase comme celle qui commence cet essai est proprement scandaleuse. Benjamin commence son essai en disant:

En aucun cas, devant une cuvre d'art ou une forme d'art, la référence au récepteur ne se révèle fructueuse pour la connaissance de cette cuvre ou de cette forme. Point ne suffit de dire que toute relation à un public déterminé ou à ses représentants détourne de la bonne voie; même le concept d'un récepteur «idéal» nuit à tous les exposés théoriques sur l'art, car ils ne sont tenus à présupposer que l'essence corporelle et spirituelle de l'homme, - dans aucune de ses cuvres il ne présuppose l'attention portée à l'homme. Aucun poeme n'est destiné au lecteur, aucun tableau au spectateur, aucune symphonie d l'auditeur.

Il ne pouvait être plus catégorique que dans cette affirmation au début de l'essai. Vous pouvez vous rendre compte comment ceci ferait souffler un petit vent de panique à Constance ${ }^{6}$, panique à laquelle ils répondraient en disant qu'il s'agit d'une théorie essentialiste de l'art,

5. Tr. M. de Gandillac, CEuvres (Paris, Denoël, 1971, p. 261). La demière phrase, omise par M. de Gandillac, est notre traduction.

6. N.d.t.: lieu d'émergence de la théorie de la réception. Jauss y enseigne. 
que cet accent mis sur l'auteur aux dépens du lecteur relève d'un pré-kantisme, puisque Kant avait déjà accordé au lecteur, au récepteur, au spectateur un rôle important, plus important que celui de l'auteur. Cette position est alors prise comme exemple d'une régression à une conception messianique de la poésie qui serait alors religieuse au mauvais sens du mot, et elle est très attaquée pour cette raison.

Mais par ailleurs Benjamin est aussi fréquemment célébré pour avoir redonné sa dimension sacrée au langage littéraire et avoir ainsi dépassé, ou du moins considérablement raffiné, l'historicité séculière sur laquelle repose la notion de modernité. Si l'on considère la modernité telle qu'elle est décrite par Gadamer comme une perte du sacré, la perte d'un certain genre d'expérience poétique, et son remplacement par un historicisme séculier qui perd le contact avec ce qui était à l'origine essentiel, on ne peut que louer Benjamin pour avoir rétabli ce contact oublié. On trouve même chez Habermas des propos en ce sens. Mais plus près de nous, nous avons l'exemple de quelqu'un qui lit Benjamin avec une grande subtilité, qui est conscient de ses complexités et qui l'apprécie précisément pour la façon dont il combine un modèle historique complexe avec un sens du sacré et c'est Geoffrey Hartman. Il écrit dans l'un de ses derniers livres:

Ce chiasme entre l'espoir et la catastrophe est ce qui sauve l'espoir de n'être démasqué que comme catastrophe: une illusion ou un mouvement de désir insatisfait qui détruit tout. Le fondement de l'espoir devient le souvenir qui sous-tend la fonction et même le devoir de l'historien et du critique. Rappeler le passé est un acte politique, une «recherche» qui nous attache à des images d'un pouvoir particulier, des images qui peuvent nous obliger à nous identifier à elles, qui en appelle à la «faible force messianique» dont nous avons $l^{\prime}$ usage ( $2^{\circ}$ thèse). Ces images, coupées de leur place fixe dans l'histoire, défont le concept de temps homogène, surgissent dans le présent ou le reconstruisent. «Ainsi, pour Robespierre, écrit Benjamin continuant les réflexions de Marx dans le 18 Brumaire, la Rome antique était un passé chargé d' «à-présent»

7. En français dans le texte. 
(Jetztzeit), surgi du continu de l'histoire. La Révolution française s'entendait comme une Rome recommencée.» (14 thèse) ${ }^{8}$

Référence est faite ici à la mémoire historique, à un concept de l'historique qui vient se rattacher, se fondre dans un concept apocalyptique, religieux, spirituel, mariant ainsi l'historique au sacré d'une manière tout à fait séduisante, attrayante. C'est en tout cas tout a fait attrayant pour Hartman et on peut le comprendre puisqu'il nous est ainsi offert à la fois le langage du désespoir, le langage du nihilisme, avec la rigueur particulière qui l'accompagne, et en même temps l'espoir! Vous avez tout en même temps: vous avez la perception critique, vous avez la possibilité de prolonger les accents apocalyptiques, vous avez cette éloquence particulière qui vient avec (on ne peut vraiment se passionner que si on écrit sur un mode apocalyptique) mais vous pouvez toujours vous exprimer en termes d'espoir et Benjamin serait un exemple de ce mélange de rigueur nihiliste et de révélation sacrée. Un esprit comme Hartman qui apprécie une perspective juste et équilibrée sur ces choses a raison de citer et d'admirer Benjamin qui en offre la possibilité. Le problème de la réception de Benjamin se fonde sur cette question du messianique et c'est très fréquemment ce texte sur «la Tâche du traducteur» qui est cité comme l'un des plus révélateurs en ce sens.

Nous poserons donc maintenant la question la plus simple, la plus naïve, la plus littérale des questions possibles au sujet du texte de Benjamin et nous n'irons pas au-delà: que dit Benjamin? Que dit-il, au niveau le plus élémentaire? Il semble absurde de poser une question si simple, qui semble si inutile tant il apparaît certain que entre personnes cultivées nous parviendrions au moins à une entente minimale sur ce qui est dit ici, nous permettant des développements à partir de cet énoncé, prendre position, le discuter, l'interpréter et ainsi de suite. Mais il semble que dans le cas de ce texte ce soit très difficile à établir. Même les traducteurs qui sont certainement proches du texte, qui ont dû le lire pour le moins attentivement, ne semblent pas avoir la moindre idée de

8. Geoffrey H. Hartman, Criticism in the Wilderness: The Study of Literature Today (New Haven, Yale University Press, 1980, p. 78). La citation de Benjamin est tirée de la traduction de M. de Gandillac. 
ce que dit Benjamin. Au point que lorsque Benjamin dit quelque chose de simple en un sens - par exemple lorsqu'il dit que quelque chose $n$ 'est pas -, les traducteurs qui connaissent au moins assez l'allemand pour savoir la différence entre quelque chose qui est et quelque chose qui $n$ 'est pas ne la font pas! et écrivent absolument et littéralement le contraire de ce que Benjamin a dit. C'est remarquable parce que les deux traducteurs que j'ai ici - Harry Zohn qui a traduit le texte en anglais et Maurice de Gandillac qui l'a traduit en français - sont de très bons traducteurs et connaissent très bien l'allemand. Harry Zohn, peut-être le connaissez-vous; Maurice de Gandillac est un éminent professeur de l'Université de Paris, homme très érudit qui connait très bien l'allemand et qui devrait savoir, par exemple, la différence entre «Ich gehe nach Paris» et «Ich gehe nicht nach Paris». Ce n'est pas plus difficile que ça mais pourtant il n'y parvient pas.

Un exemple qui est devenu fameux et qui a une histoire se trouve dans le passage situé à la fin de l'essai de Benjamin, où Benjamin dit la chose suivante: «Wo der Text unmittelbar, ohne vermitelnden Sinn», etc., «der Wahrheit oder der Lehre angehört, ist er übersetzbar schlechthin» (62): «Là où le texte appartient directement, sans médiation, au domaine de la vérité et du dogme, il est, sans problèmes, traduisible» - le texte peut être traduit, schlechthin, il n'y a donc pas de problème pour le traduire. Gandillac - je n'en donnerai pas de commentaires - traduit cette phrase simple, énonciatoire: «Là où le texte, immédiatement, sans l'entremise d'un sens ... relève de la vérité ou de la doctrine, il est purement et simplement intraduisible» (275) - in-traduisible. Ce qui ajoute une touche d'humour à cet exemple-là, c'est que Jacques Derrida donnait un séminaire sur ce texte à Paris, en utilisant la version française - l'allemand de Derrida est plutôt bon, mais il préfere utiliser la version française et quand on est philosophe en France on prend Gandillac plutôt au sérieux. Derrida consacrait donc une partie de sa lecture à l'«intraduisible», à l'intraduisibilité, lorsque quelqu'un du séminaire (m'a-t-on dit) lui fit remarquer que le terme correct était «traduisible». Je suis sûr que Derrida a pu expliquer qu'il s'agissait de la même chose - et je le dis positivement: $c$ 'est la même chose, mais, cependant, ce n'est pas la même chose sans une explication supplémentaire. Il s'agit là d'un exemple et nous en verrons bientôt d'autres qui se rapportent davantage aux questions que nous allons soulever à propos de ce texte. 
Pourquoi, d'abord, le traducteur est-il dans ce texte la figure exemplaire? Pourquoi le traducteur est-il cité en exemple par rapport aux questions très générales concernant la nature du langage poétique posées par le texte? Le texte est une poétique, une théorie du langage poétique, pourquoi alors Benjamin ne traite-t-il pas des poètes? ou du lecteur, éventuellement, ou du couple poète-lecteur comme dans le modèle de la théorie de la réception? Et puisque, de toute façon, il est si négatif sur la notion de réception, qu'est-ce qui fait, de manière significative, la différence entre le couple auteur-lecteur et le couple auteur-traducteur, dans la mesure où, à première vue, le traducteur apparaît simplement comme un lecteur du texte original? On peut donner, jusqu'à un certain point, des réponses empiriques assez évidentes. L'essai a été écrit, comme vous le savez, en introduction à la propre traduction faite par Benjamin des Tableaux parisiens de Baudelaire: il aurait pu choisir la figure du traducteur juste par mégalomanie. Mais ce n'est pas le cas. L'une des raisons pour lesquelles il choisit le traducteur plutôt que le poète est que le traducteur, par définition, échoue. Le traducteur ne pourra jamais faire ce que le texte original a fait. Toute traduction est toujours seconde par rapport à l'original et le traducteur en tant que tel a perdu dès le tout début. Il est par définition sous-payé, il est par définition débordé par la tâche, il est par définition celui que l'histoire ne retiendra pas vraiment comme un égal, à moins qu'il ne soit aussi poète, mais ce n'est pas toujours le cas. Si le texte s'intitule «Die Aufgabe des Übersetzers», il nous faut lire ce titre plus ou moins comme une tautologie: Aufgabe, la tâche, peut aussi signifier celui qui doit abandonner. Si vous entrez dans le Tour de France et que vous abandonnez, ce sera la Aufgabe: «er hat aufgegeben», il ne continue plus la course. C'est aussi, en ce sens, la défaite, l'abandon du traducteur. Le traducteur doit abandonner pour ce qui est de la tâche de retrouver ce qui était dans l'original.

La question est alors de savoir pourquoi cet échec en ce qui concerne un texte original, un poète original, est pour Benjamin exemplaire? La question devient aussi en quoi le traducteur est-il différent du poète? Et sur ce point Benjamin affirme catégoriquement que le traducteur est radicalement différent, qu'il differe essentiellement du poète et de l'artiste. C'est une curieuse chose à dire, une chose qui va à l'encontre du sens commun car on supposerait (et à l'évidence 
c'est le cas) que certaines des qualités nécessaires à un bon traducteur sont similaires aux qualités nécessaires à un bon poète. Ce qui ne veut pas dire qu'ils font la même chose. L'affirmation est si frappante, si choquante en un sens, que là aussi le traducteur (Maurice de Gandillac) ne la voit pas. Benjamin dit (dans la traduction de Zohn): «Although translation, unlike art, cannot claim permanence for its products...» (75). Gandillac, pour le même passage: «Ainsi la traduction, encore qu'elle ne puisse élever une prétention à la durée de ses ouvrages, et en cela elle $n$ 'est pas sans ressemblance avec l'art...» (267). L'original est sans ambiguïté aucune: «Übersetzung also, wiewohl sie auf Daueur ihrer Gebilde nicht Anspruch erheben kan und hierin unähnlich der Kunst...» (55) Lorsque vous tombez dessus dans un texte, le propos est si surprenant, va tellement contre le sens commun, qu'un traducteur intelligent, érudit et prudent ne peut pas le voir, ne peut pas voir ce que Benjamin dit. C'est remarquable. Zohn l'a vu - n'allez pas croire que Zohn a tout compris et Gandillac rien du tout: d'une manière générale, en dernière analyse, je pense que Gandillac va un peu plus loin que Zohn.

Quoi qu'il en soit, Benjamin établit une franche distinction entre eux. Il n'est pas nécessaire pour de bons traducteurs d'être de bons poètes. Certains des meilleurs traducteurs - il mentionne Voss (traducteur de Homère), Luther et Schlegel - sont des poètes médiocres. Il existe des poètes qui sont aussi des traducteurs: il mentionne Holderlin qui traduisit Sophocle et d'autres auteurs, et George qui traduisit Baudelaire - Dante également mais surtout Baudelaire, ce qui rapproche Benjamin de George. Cependant, dit-il, ce n'est pas parce que ce sont de grands poètes que ce sont de grands traducteurs, ce sont de grands poètes et de grands traducteurs. Ce ne sont pas simplement, comme Heidegger le dira de Hölderlin, des Dichter der Dichter, mais ce sont des Übersetzer dè Dichter, ils sont au-dessus des poètes parce qu'ils sont aussi traducteurs.

Une série des plus grands, comme Luther, Voss, Schlegel, sont comme traducteurs incomparablement plus importants que comme écrivains; d'autres, parmi les plus grands de tous, comme Hölderlin et George, si l'on considère l'ensemble de leur œuvre créatrice, ne doivent pas être considérés seulement comme des écrivains, [et notamment pas si nous les 
considérons comme des traducteurs]. En effet, de même que la traduction est une forme propre, on peut envisager la tâche du traducteur aussi comme une tâche propre et la distinguer avec précision de celle de l'écrivain. (269)

Des différences existant entre la situation du traducteur et celle du poète, la première qui vient à l'esprit est que le poète entretient un rapport avec la signification, avec un énoncé qui n'est pas entièrement du domaine du langage. C'est là la naîveté du poète: qu'il a quelque chose à dire, qu'il doit transmettre une signification qui n'est pas nécessairement en relation avec le langage. Le rapport du traducteur à l'original est le rapport entre langage et langage, dans lequel le problème de la signification ou du désir de dire quelque chose, le besoin d'affirmer quelque chose sont entièrement absents. La traduction est un rapport de langage à langage, pas un rapport à une signification extralinguistique, qui peut être copié, paraphrasé ou imité. Ce n'est pas le cas du poète: la poésie n'est certainement pas paraphrase, clarification ou interprétation, une copie en un sens. Voici déjà une première différence.

Si elle n'est pas fondamentalement comme la poésie, alors à quoi la traduction ressemble-t-elle dans le texte de Benjamin? L'une des choses à laquelle elle ressemble serait la philosophie en ce qu'elle est critique, en ce que la philosophie est critique de la simple notion d'imitation, du discours philosophique comme Abbild (imitation, paraphrase, reproduction) de la situation réelle. La philosophie n'est pas dans un rapport d'imitation au monde tel que nous le connaissons mais entretient une autre relation avec ce monde. La philosophie critique et référence est faite de nouveau spécifiquement à Kant - sera de la même manière critique de la notion d'un concept imitatif du monde.

Um das echte Verhältnis zwischen Original und Übersetzung zu erfassen, ist eine Erwăgung anzustellen, deren Absicht durchauss den Gedankengången analog ist, in denen die Erkenntniskritik die Ünmőglichkeit einer Abbildstheorie zu erweisen hat. (53)

9. La phrase entre crochets n'est pas traduite par M. de Gandillac. 
Pour saisir le rapport authentique entre original et traduction, il faut procéder à un examen dont le propos est tout à fait analogue aux démarches de la pensée par lesquelles la critique de la connaissance doit démontrer l'impossibilité de la théorie de l'image-copie. ${ }^{10}$

Kant serait effectivement critique de la notion d'art comme imitation. Cela serait vrai aussi de Hegel dans une certaine mesure puisqu'il y a précisément un élément critique qui intervient et qui fait disparaître cette image, ce modèle, qui détruit, annule ce concept d'imitation.

La traduction, dit Benjamin, est aussi davantage comme la critique ou la théorie littéraire que comme la poésie même. C'est en se situant lui-même par rapport à Friedrich Schlegel et au romantisme allemand en général que Benjamin établit cette similarité entre la critique littéraire (au sens de théorie de la littérature) et la traduction. Et cette référence historique ici au romantisme de Iéna donne aux notions de critique et théorie littéraires une dignité qu'habituellement elles ne possèdent pas forcément. La critique comme la traduction sont toutes deux prises dans un mouvement que Benjamin appelle ironique, un mouvement qui défait la stabilité de l'original en lui donnant dans la traduction ou la théorisation une forme définitive, canonique. Curieusement, la traduction canonise son propre texte plus que l'original n'était canonique. Que l'original ne soit pas entièrement canonique apparait clairement du fait qu'il appelle une traduction, il ne pouvait être définitif puisqu'il peut être traduit. Mais, dit Benjamin, vous ne pouvez traduire la traduction; lorsque que vous avez une traduction, vous ne pouvez la traduire encore une fois. Vous ne pouvez traduire qu'un original. La traduction canonise, fige l'original et révèle dans l'original une mobilité, une instabilité qu'on ne remarquait pas d'emblée. L'acte de lecture critique, théorique, accompli par un critique comme Friedrich Schlegel et par la théorie littéraire en général, par lequel l'œuvre originale n'est pas imitée ou reproduite mais d'une certaine manière mise en mouvement, dé-canonisée, interrogée d'une

10. Tr. de M. de Gandillac, p. 265. Dans sa traduction, de Man ajoute après «critique de la connaissance» qu'il traduit «critical epistemology»: «voici Kant, Erkenntniskritik». 
façon qui ruine sa prétention à l'autorité canonique, est semblable à l'action du traducteur.

Enfin, la traduction est comme l'histoire et ce sera la chose la plus difficile à comprendre. Dans ce qui est le passage le plus difficile de ce texte, Benjamin dit qu'elle est comme l'histoire dans la mesure où l'histoire ne doit pas être comprise par analogie avec un quelconque processus naturel. Nous ne devons pas penser l'histoire comme une maturation, une croissance organique, ou même comme une dialectique, quoi que ce soit qui ressemble à un processus naturel de croissance et de mouvement. Il nous faut penser l'histoire à l'inverse: il nous faut comprendre les changements naturels du point de vue de l'histoire plutôt que comprendre l'histoire du point de vue des changements naturels. Si nous voulons comprendre ce qu'est la maturation, nous devrions le comprendre du point de vue du changement historique. De la même manière, la relation entre la traduction et l'original ne doit pas être comprise par analogie avec les processus naturels tels que la ressemblance ou la dérivation par analogie formelle; nous devons plutôt comprendre l'original du point de vue de la traduction. Comprendre ce schéma historique est la fonction attachée à toute lecture de ce texte-ci.

Toutes les activités qui ont été mentionnées - philosophie comme épistémologie critique, critique et théories littéraires (à la façon de Friedrich Schlegel) ou histoire comprise comme processus non-organique - sont elles-mêmes toutes dérivées d'activités originales. La philosophie est un dérivé de la perception mais elle n'est pas comme la perception car elle est l'examen critique des prétentions à la vérité de la perception. La critique est un dérivé de la poésie parce qu'elle est inconcevable sans la poésie qui la précède. L'histoire est un dérivé de l'action pure puisqu'elle vient nécessairement à la suite d'actions qui ont déjà eu lieu. Dans la mesure où toutes ces activités sont dérivées d'activités originales, elles sont singulièrement inachevées, elles ont échoué, elles sont, dans un sens, avortées dès le début puisqu'elles sont dérivées et secondes. Et pourtant Benjamin insiste sur le fait que le modèle de leur dérivation n'est pas celui de la ressemblance ou de l'imitation. Ce n'est pas un processus naturel: la traduction ne ressemble pas à l'original de la manière dont un enfant ressemble aux parents; ce n'est pas non plus une imitation, une copie ou une paraphrase de l'original. En ce sens, puisque ce ne sont pas des ressemblances, des 
imitations, on serait tenté de dire que ce ne sont pas des métaphores. La traduction n'est pas la métaphore de l'original. Néanmoins le mot allemand pour traduction, übersetzen, signifie métaphore. Übersetzen traduit exactement le grec meta-phorein: transporter au-delà, übersetzen: mettre de l'autre côté. Übersetzen, devrais-je dire, traduit métaphore, ce qui, affirme Benjamin, n'est pas du tout la même chose. Ce ne sont pas des métaphores mais le mot signifie métaphore. La métaphore n'est pas une métaphore, c'est ce que dit Benjamin. Il n'est pas étonnant que les traducteurs éprouvent des difficultés. C'est une curieuse affirmation que de dire que übersetzen n'est pas métaphorique, que übersetzen ne se fonde pas sur la ressemblance, qu'il n'y a pas de ressemblance entre la traduction et l'original. Propos étonnamment paradoxal: la métaphore n'est pas métaphore.

Toutes ces activités - philosophie critique, théorie littéraire, histoire - se ressemblent en ce qu'elles ne ressemblent pas à ce dont elles sont dérivées. Mais elles sont toutes interlinguistiques: elles se rapportent à ce qui dans l'original relève du langage et non à la signification entendue comme un corrélat extralinguistique susceptible de paraphrase et d'imitation. Elles désarticulent, elles défont l'original, elles révèlent que l'original était déjà et depuis toujours désarticulé. Elles révèlent que leur échec qui semble être dû au fait qu'elles sont secondes par rapport à l'original révèle un échec essentiel, une désarticulation essentielle qui était déjà là dans l'original. Elles tuent l'original en dévoilant que l'original était déjà mort. Elles lisent l'original du point de vue d'un pur langage (reine Sprache), un langage qui serait entièrement libéré de l'illusion de la signification, une forme pure si vous voulez. Et ce faisant, elles mettent au jour un démantèlement, une dé-canonisation qui était déjà là dans l'original, depuis le début. Dans le processus de traduction tel que Benjamin le comprend - qui a peu à voir avec l'action empirique de traduction que nous pratiquons tous quotidiennement - il existe un danger intrinsèque et particulièrement menaçant. La traduction qu'a faite Hoblderlin de Sophocle est emblématique de ce danger.

A cet égard, comme de tout autre point de vue essentiel, les traductions de Hőlderlin, surtout celle des deux tragédies de Sophocle, représentent une confirmation de notre thèse. L'harmonie entre les langues y est si profonde que le sens 
n'est touché par le vent du langage qu'à la manière d'une harpe éolienne. (...) Et c'est précisément pourquoi elles sont exposées plus que d'autres à l'immense danger qui, dès le départ, guette toute traduction: que les portes d'un langage si élargi et si dominé retombent et enferment le traducteur dans le silence. Ses traductions de Sophocle furent les dernières œuvres de Hölderlin. Ici le sens s'effondre d'abîme en abîme, jusqu'à risquer de se perdre dans les gouffres sans fond du langage. (275)

La traduction, dans la mesure où elle désarticule l'original, dans la mesure où elle est pur langage et n'est concernée que par le langage, est entraînée dans ce qu'il appelle l'abîme sans fond, quelque chose d'essentiellement destructeur qui se trouve dans le langage lui-même.

Ce que fait la traduction, par rapport à la fiction ou à l'hypothèse d'un pur langage libéré du poids de la signification, est qu'elle suggère, en amenant au jour ce que Benjamin appelle «die Wehen des eigerıen» - la souffrance de ce qu'on pense nous être particulier - , la souffrance du langage original. Nous pensons être à l'aise dans notre propre langage, nous ressentons un confort, une familiarité, un abri dans le langage que nous appelons nôtre, dans lequel nous pensons ne pas être aliénés. Ce que révèle la traduction, c'est que cette aliénation est la plus forte dans notre relation à notre propre langage original, c'est que le langage original dans lequel nous sommes impliqués est désarticulé d'une façon qui nous impose une aliénation particulière, une douleur particulière. Là aussi, les traducteurs, à l'unisson, ne peuvent pas voir ce qui est dit. Le texte de Benjamin est le suivant: «...dass gerade unter allen Formen ihr als Eigenstes es zufallt, auf jene Nachreife des fremden Wortes, auf die Wehen des eigenen zu merken» (54). Les deux traducteurs - je pense qu'ils ne se sont pas consultés, ils l'ont fait $d^{\prime}$ 'un commun accord ${ }^{11}$ - traduisent Wehen, douleurs, les «douleurs de l'enfantement», celles qui désignent spécifiquement les souffrances attachées à l'accouchement. Gandillac est très explicite sur ce point, il les appelle «les douleurs obstétricales» (266) au sens le plus littéral, le plus clinique; Zohn dit «birth pangs»

11. En français dans le texte. 
(73). Pourquoi ils le font est un mystère. Wehen peut signifier les douleurs de l'enfantement mais le mot désigne toutes sortes de souffrances, sans nécessairement connoter naissance et renaissance, résurrection, qui serait associée à la notion de douleurs d'enfantement parce que vous souffrez en produisant quelque chose - et c'est un moment magnifique, vous voudriez cette souffrance (c'est particulièrement facile pour nous de le dire). Benjamin ne parlait que de «Nachreife des fremden Wortes», traduit par Zohn «maturing process» [processus de maturation], ce qui est également faux. Nachreife est semblable au mot allemand Spätlese (un vin particulièrement bon fait des raisins tardifs, gâtés), c'est comme le roman de Stifter Nachsommer («l'Été indien»), on y trouve la mélancolie, le sentiment d'un léger épuisement, d'une vie à laquelle vous n'avez pas droit, un bonheur auquel vous n'avez pas droit, le temps qui a passé, et ainsi de suite. Il $\mathrm{y}$ a un lien avec un autre mot que Benjamin utilise constamment, le mot überleben, vivre au-delà de votre propre mort en quelque sorte. La traduction n'appartient pas à l'original, l'original est déjà mort mais la traduction appartient à l'après-vie de l'original, supposant et confirmant ainsi la mort de l'original. Nachreife est du même ordre ou relève de la même chose. Ce n'est d'aucune manière un processus de maturation, c'est un regard en arrière sur un processus de maturation qui est achevé et qui n'a plus lieu. Si vous traduisez donc Wehen par «douleurs de l'enfantement», vous devriez autant le traduire par «douleurs de la mort» que par «douleurs de l'enfantement» et l'accent est peut-être davantage sur la mort que sur la vie.

Le processus de traduction, si nous pouvons l'appeler un processus, en est un de changement et de mouvement qui a l'apparence de la vie mais de la vie comme après-vie parce que la traduction révèle aussi la mort de l'original. Pourquoi en est-il ainsi? Quelles sont ces douleurs de mort, peut-être d'enfantement, de l'original? Il est facile de dire dans une certaine mesure ce que cette douleur n'est pas. Il ne s'agit certainement pas de souffrances subjectives, une sorte de pathos du soi, une sorte de manifestation d'auto-pathos que le poète exprimerait comme sa souffrance. Ce n'est certainement pas le cas parce que, dit Benjamin, les souffrances ici mentionnées ne sont d'aucune manière humaines. Elles ne sont certainement pas celles d'un individu ou d'un sujet. Cela est également très difficile à voir pour les traducteurs. Zohn, devant ce passage (je vais arrêter de jouer à monter en épingle les 
traductions mais cela est toujours intéressant), traduit: «if they are referred exclusively to man» (70) [si elles sont rapportées exclusivement à l'homme]. Benjamin dit très clairement: «wenn sie nicht... auf den Menschen bezogen werden» (51): si vous ne les mettez pas en rapport avec l'homme. Il insiste précisément sur le fait que la souffrance mentionnée, l'échec, n'est pas un échec de l'homme, cela ne renvoie donc aucunement à une quelconque expérience subjective. L'original est sans ambiguité à cet égard. La souffrance n'est pas non plus une sorte de pathos historique, le pathos évoqué par Hartman lorsqu'il attribue à Benjamin la découverte du pathos de l'histoire. Ce n'est pas ce pathos du souvenir ou ce mélange émouvant d'espoir, de catastrophe et d'apocalypse que cerne Hartman et qui est certainement présent dans le style de Benjamin mais pas tant dans ce qu'il dit. Ce n'est pas le pathos d'une histoire, ce n'est pas le pathos de ce qui chez Hölderlin est nommé le «dürftige Zeit», entre la disparition des dieux et leur éventuel retour. Ce n'est pas cette sorte de geste sacrificiel, dialectique et élégiaque, au moyen duquel on regarde le passé d'une période qui n'est plus et qui vous donne alors l'espoir d'un autre futur qui pourrait advenir.

Les raisons de ce pathos, de ces Wehen, de cette souffrance sont spécifiquement linguistiques. Benjamin les avance dans une perspective linguistique avec une remarquable précision structurale. Ainsi lorsque vous trouvez le mot "abîme» dans le passage sur Hőlderlin, où il est dit que Hőlderlin chute dans l'abîme du langage, il faut comprendre le mot «abîme» non dans un sens de pathos mais dans son acception technique: comme lorsque vous parlez d'une structure de mise en abyme, ${ }^{12}$ le genre de structure qui fait apparaître clairement que le texte lui-même est un exemple de ce qu'il démontre. Le texte sur la traduction est lui-même une traduction et l'intraduisibilité qu'il mentionne à son propre endroit est saisie dans sa propre texture et se saisira de quiconque tentera à son tour de le traduire, comme je le fais maintenant, en n'y parvenant pas. Le texte est intraduisible, il était intraduisible pour les traducteurs qui ont tenté de le faire, il est intraduisible pour les commentateurs qui en parlent, il est exemplaire de

12. En français dans le texte. 
ce qu'il avance, c'est une mise en abyme au sens technique, un récit à l'intérieur du récit de ce qu'il propose.

Quelles sont les raisons linguistiques qui poussent Benjamin à parler d'une souffrance, d'une désarticulation, d'une désintégration de toute cuvre originale ou de toute cuvre pour autant que ce soit une œuvre de langage? Benjamin est très précis sur ce point et nous offre en quelques lignes ce qui se révèle être une complète théorie du langage. La disjonction opère d'abord entre ce qu'il appelle «das Gemeinte», ce qui est signifié, et «die Art des Meinens», la manière dont le langage signifie, entre logos et lexis si vous voulez - ce qu'une certaine proposition signifie et la manière par laquelle la proposition est signifiée. Ici les difficultés des traducteurs sont un peu plus intéressantes car elles impliquent des concepts philosophiques importants. Gandillac est un philosophe qui connaît la phénoménologie et qui écrit dans une période où la phénoménologie domine le terrain philosophique en France; il traduit: «visée intentionnelle» (272). La manière dont nous traduirions aujourd'hui en français «das Gemeinte» et "Art des Meinens» serait de faire la distinction entre vouloir dire et dire ${ }^{13}$. Zohn traduit par «the intended object» [l'objet intentionné] et «the mode of intention» [le mode d'intentionnalité] (74). Nous sommes ici en présence d'un présupposé phénoménologique et Gandillac fait référence à Husserl dans une note. Les deux traducteurs posent que le sens et la manière par laquelle le sens est produit sont des actes intentionnels. Mais le problème est précisément que si la fonction de signification est certainement intentionnelle, il n'est pas certain a priori que le mode de signification, la manière par laquelle je signifie, est d'une quelconque manière intentionnelle. La manière par laquelle je peux essayer de signifier dépend de propriétés linguistiques qui ne sont pas uniquement faites par moi car je dépends, pour utiliser ses facultés, du langage tel qu'il existe, il n'est pas fait en tant que tel par nous en tant qu'êtres historiques, il n'est peut-être même pas du tout fait par des humains. Benjamin dit dès le début qu'il n'est pas certain du tout que le langage soit d'aucune façon humain. Poser l'équation entre le langage et l'humanité - ce que fit Schiller, comme nous l'avons vu hier - est problématique. Si le langage n'est pas nécessairement humain - si

13. En français dans le texte. 
nous obéissons à la loi, si nous fonctionnons à l'intérieur du langage et uniquement en termes de langage - , il ne peut pas y avoir d'intention. Il peut $y$ avoir une intention de signification mais il n'y a pas d'intention au sens purement formel en ce que nous utilisons le langage indépendamment du sens ou de la signification. La traduction qui situe l'intentionnalité des deux côtés, dans l'acte de signification et dans la manière dont on signifie, se trompe sur un point philosophique important, car ce qui est en jeu est la possibilité d'une phénoménologie du langage, ou d'un langage poétique, la possibilité d'établir une poétique qui serait à tous points de vue une phénoménologie du langage.

Comment comprendre cette différence entre "das Gemeinte» et «Art des Meinens», entre dire et vouloir dire? Benjamin donne 1'exemple du mot allemand Brot et du mot français pain. Pour signifier le pain, lorsque j'ai besoin de désigner le pain, j'ai le mot Brot, de sorte que ma manière de le signifier est d'utiliser le mot Brot. La traduction va révéler une différence fondamentale entre l'intention de désigner Brot et le mot Brot même, dans sa matérialité, comme un moyen de signification. Si vous entendez Brot dans ce contexte de Hölderlin qui est si souvent mentionné dans ce texte, j'entends nécessairement $B r o t$ und Wein qui est le grand texte de Hölderlin très présent ici. Ce qui en français devient Pain et vin. «Pain et vin», c'est ce que vous avez gratuitement dans un restaurant, dans un restaurant bon marché où ils sont encore compris dans le prix, de sorte que pain et vin a des connotations très différentes de Brot und Wein. Il évoque pain français, baguette, ficelle, batard, toutes ces choses - j'entends maintenant «bastard» [bâtard] dans Brot. Cela dérange la stabilité du quotidien. J'étais très heureux avec le mot Brot, qui pour moi a une résonnance familière car ma langue maternelle est le flamand et nous disons brood, comme en allemand, mais si je dois penser que Brot [brood] et pain sont la même chose, ça me trouble terriblement. Ça va en anglais parce que «bread» est assez proche de Brot [brood], malgré l'usage idiomatique de «bread» pour l'argent qui pose ses problèmes. Mais la stabilité de mon quotidien, de mon pain quotidien, les aspects quotidiens rassurants du mot «bread», le pain quotidien, cela est troublé par le mot français pain. Ce que je signifie est troublé par la manière dont je signifie - la manière dont c'est du pain, le phonème, le terme pain, 
avec un ensemble de connotations qui vous entraîne dans une direction complètement différente.

Cette disjonction sera mieux comprise (en la déplaçant vers un problème théorique plus familier) si on la pose dans les termes du rapport difficile entre l'herméneutique et la poétique de la littérature. Lorsque vous faites de l'herméneutique, vous traitez du sens de l'œuvre; lorsque vous faites de la poétique, vous traitez de stylistique ou vous décrivez la manière dont une cuvre signifie. La question est de savoir si ces deux démarches sont complémentaires, si vous pouvez rendre compte de toute l'œuvre en faisant en même temps de l'herméneutique et de la poétique. L'expérience montre que ce n'est pas le cas. Lorsqu'on essaye de parvenir à cette complémentarité, la poétique est toujours perdante et ce qu'on fait toujours, c'est de l'herméneutique. Les problèmes de signification sont si prenants qu'il est impossible de faire de l'herméneutique et de la poétique en même temps. Dès que vous commencez à traiter des problèmes de signification, comme j'ai malheureusement tendance à le faire, la poétique est négligée. Les deux ne sont pas complémentaires, les deux peuvent être, d'une certaine manière, exclusives l'une de l'autre et cela fait partie du problème posé par Benjamin, un problème purement linguistique.

Il en donne encore un développement quand il parle d'une disjonction entre le mot et la phrase, Wort et Satz. Satz en allemand ne signifie pas simplement la phrase au sens grammatical, il signifie le principe: Heidegger emploiera Der Satz vom Grund [le principe de raison]. Satz, c'est le principe, la proposition la plus fondamentale, le sens - le mot le plus signifiant - alors que le mot est associé chez Benjamin avec Aussage, la manière dont vous énoncez la proposition, l'agent apparent de la proposition. Wort ne signifie pas uniquement l'agent de la proposition en tant qu'unité lexicale mais aussi comme syntaxe et comme grammaire. Si vous considérez une phrase en termes de mots, vous ne la considérez pas simplement en termes de mots particuliers mais aussi dans la perspective des rapports grammaticaux établis entre ces mots. Ainsi la question de la relation entre le mot et la phrase devient pour Benjamin la question de la compatibilité entre grammaire et sens. Ce qui est remis en question est précisément cette compatibilité que nous tenons pour acquise dans toutes sortes de réflexions linguistiques. La grammaire (mot et syntaxe) d'un côté et le 
sens (tel qu'il culmine dans le Satz) de l'autre sont-ils compatibles l'un avec l'autre? Est-ce que l'un mène à l'autre, est-ce que l'un soutient l'autre? Benjamin nous dit que la traduction remet en question cette croyance parce que, dit-il, dès qu'une traduction est véritablement littérale, wörtlich, mot-à-mot, le sens disparaît complètement. L'exemple est de nouveau les traductions de Sophocle par Holderlin qui sont absclument littérales, mot-à-mot, et qui en sont totalement incompréhensibles. Ce qui ressort est complètement incompréhensible et défait complètement la phrase, le Satz de Sophocle, qui a entièrement disparu. Le sens du mot s'efface (comme nous l'avons vu, un mot comme Aufgabe, qui signifie «tâche», signifie aussi quelque chose de complètement différent, de sorte que le mot nous échappe) et il n'y a pas de moyen grammatical pour contrôler cet effacement. Il y a aussi effacement total du sens lorsque le traducteur suit la syntaxe, lorsqu'il écrit littéralement, wörtlich. Et jusqu'à un certain point, un traducteur doit être wörtlich, doit être littéral. Le problème se compare parfaitement à la relation entre la lettre et le mot; la relation entre le mot et la phrase est comme la relation entre la lettre et le mot, c'est-à-dire que la lettre, par rapport au mot, n'a pas de sens, elle est a-semos, elle n'a pas de sens. Lorsque vous épelez un mot, vous dites un certain nombre de lettres sans signification qui s'assemblent ensuite dans le mot mais dans chacune des lettres le mot est absent. La lettre et le mot sont absolument indépendants l'un de l'autre. Ce qui est désigné ici comme la disjonction entre la grammaire et le sens, Wort et Satz, c'est la matérialité de la lettre: l'indépendance ou la manière dont la lettre peut déranger l'apparente stabilité du sens d'une phrase et y introduire un glissement qui fera disparaître ce sens, le fera devenir évanescent, et qui fera perdre tout contrôle sur ce sens.

Nous avons donc premierement une disjonction dans le langage entre l'herméneutique et la poétique, nous en avons une deuxième entre la grammaire et le sens et nous aurions enfin une disjonction, selon Benjamin, entre le symbole et ce qui est symbolisé, une disjonction au niveau des tropes entre le trope en tant que tel et le sens comme pouvoir totalisant de substitutions tropologiques. Il y a une disjonction similaire et aussi radicale entre ce que les tropes (qui suggèrent toujours une totalisation) véhiculent en termes de totalisation et ce que les tropes réalisent par eux-mêmes. Cela semble être la principale difficulté de ce texte-ci parce qu'il est rempli de tropes et qu'il choisit des tropes qui 
véhiculent l'illusion de totalité. Il semble retomber dans les erreurs tropologiques qu'il dénonce. Le texte utilise constamment des images de noyau, de maturation, d'harmonie, il utilise l'image de l'écorce et [du] noyau ${ }^{14}$, qui semblent dériver d'analogies entre la nature et le langage alors qu'il est constamment affirmé que de telles analogies n'existent pas. De même que l'histoire ne doit pas être comprise en termes d'analogie avec la nature, les tropes ne devraient pas être fondées sur des ressemblances avec la nature. Mais c'est précisément la difficulté et le défi de ce texte-ci. Chaque fois que Benjamin utilise un trope qui semble véhiculer une signification complète, une totale adéquation entre la figure [rhétorique] et le sens, une parfaite figure de synecdoque dans laquelle le trope partiel exprime la totalité d'un sens, Benjamin manipule le contexte allusif à l'intérieur de son texte afin que le symbole traditionnel soit déplacé de telle sorte qu'il montre la différence entre le symbole et le sens plutôt que leur accord.

Un exemple frappant est celui de l'image de l'amphore:

Car, de même que les débris d'une amphore, pour qu'on puisse restituer le tout, doivent être contigus dans les plus petits détails, mais non identiques les uns aux autres, ainsi, au lieu de se rendre semblable au sens de l'original, la traduction doit bien plutôt, dans un mouvement d'amour et jusque dans le détail, faire passer dans sa propre langue le mode de visée de l'original: ainsi, de même que les débris deviennent reconnaissables comme fragments d'une même amphore, original et traductions deviennent reconnaissables comme fragments d'un langage plus grand. Et c'est bien pourquoi la traduction ne peut que renoncer au projet de rien communiquer [...]. (271-272)

Selon cette image, il existe une langue pure, originale dont toute cuvre particulière n'est qu'un fragment. Cela serait parfait si nous pouvions, par ce fragment, avoir de nouveau accès à l'œuvre originale. L'image est celle d'un vase dont l'œuvre littéraire serait un morceau puis la traduction un morceau de l'cuvre. Il est admis que la traduction est un

14. En français dans le texte. 
fragment mais si la traduction se rapporte à l'original comme le fait un fragment, si la traduction en tant que telle reconstituait l'original, alors - bien qu'elle ne lui ressemble pas mais lui corresponde parfaitement (comme dans le mot symbolon, qui suppose la correspondance de deux morceaux ou fragments) - nous pouvons considérer toute auvre comme un fragment de la langue pure et la proposition de Benjamin serait de fait une proposition religieuse sur l'unité fondamentale des langages.

Mais Benjamin nous a dit que le symbole et ce qu'il symbolise, le trope et ce qu'il représente, ne correspondent pas. Comment concilier cela avec une proposition comme celle que nous trouvons ici? Un article de Carol Jacobs, paru dans Modern Language Notes, intitule «The Monstrosity of Translation» rend compte de ce passage d'une façon qui me semble tout à fait précise et correcte. En premier lieu elle a conscience du sens kabbalistique du texte et elle renvoie à Gershom Scholem qui, à propos de ce texte, met en rapport la figure de l'ange et l'histoire du tikkun de la kabbale lourianique:

Cependant Benjamin pense aussi au concept kabbalistique de tikkun, la restauration et réparation messianique qui rassemble et restaure l'être original des choses, brisées et souillées dans la «brisure des vases», de même que [l'être original] de l'histoire.

Et Carol Jacobs fait ce commentaire:

Scholem aurait pu citer «Die Aufgabe des Übersetzers» dans lequel l'image du récipient brisé joue un rôle plus direct. (...) Cependant, alors que Zohn suggère qu'une totalité de fragments sont réunis, Benjamin insiste sur le fait que le résultat final est toujours «un morceau brisé». ${ }^{15}$ (763, note 9)

Pour voir cela, tout ce que vous avez à faire est de traduire correctement, au lieu de traduire comme Zohn, qui rend ce passage difficile très clair mais qui, ce faisant, lui fait dire quelque chose de

15. Carol Jacobs, «The Monstrosity of Translation», Modern Language Notes, v. 90 (1975), p. 763, n. 9. 
complètement différent. Zohn dit: «des fragments d'un vase qui doivent être collés ensemble doivent se correspondre dans le plus petit détail.» Benjamin dit, selon la traduction de Carol Jacobs, mot-à-mot: «des fragments d'un récipient, afin d'être articulés ensemble» - ce qui est mieux qu'être «collés ensemble», qui présente un aspect concret totalement inapproprié - «doivent se suivre dans le plus petit détail» - ce qui n'est pas du tout la même chose que «se correspondre». La différence est déjà que nous avons folgen et non pas gleichen, non pas «correspondre». Nous avons une structure métonymique, une structure en continuité où les choses se suivent plutôt qu'une structure métaphorique et unifiante où les choses se fondent par ressemblance. Elles ne se correspondent pas, elles se suivent; elles sont déjà des métonymies et non des métaphores. En tant que telles, elles travaillent certainement moins vers une séduisante totalisation tropologique que si nous utilisions le terme «se correspondre».

Mais les choses deviennent encore plus compliquées, ou plus déformées, dans ce qui suit:

Ainsi, au lieu de se rendre similaire au sens, au Sinn de l'original, la traduction doit plutôt, avec amour et dans les détails, dans son propre langage, se former selon la manière de signifier (Art des Meinens) de l'original, afin de les rendre tous deux reconnaissables comme les morceaux brisés du langage plus grand, de même que des fragments sont les morceaux brisés d'un récipient.

Cela est entièrement différent de ce que dit Zohn [ou Gandillac ${ }^{16}$ ]:

(...) ainsi, au lieu de se rendre semblable au sens de l'original, la traduction doit bien plutôt, dans un mouvement d'amour et jusque dans le détail, faire passer dans sa propre langue le mode de visée de l'original: ainsi, de même que les débris deviennent reconnaissables comme fragments d'une même

16. N.d.t.: nous donnons ici le passage dans la traduction de Gandillac dans la mesure où celui-ci commet la même «erreur» que Zohn. 
amphore, original et traductions deviennent reconnaissables comme fragments d'un langage plus grand. (271-272)

«De même que les débris deviennent reconnaissables comme fragments d'une même amphore» est une synecdoque. «De même que des fragments, dit Benjamin, sont les morceaux brisés d'un vase»: il ne dit donc pas que les fragments constituent une totalité, il dit que les fragments sont des fragments et qu'ils resteront essentiellement fragmentaires. Ils se suivent, métonymiquement, et ils ne constitueront jamais une totalité. Cela me rappelle un exemple donné par le philosophe français Michel Serres: vous découvrez ce que sont les fragments en faisant la vaisselle; si vous cassez une assiette, elle se brise en fragments mais vous ne pouvez pas briser les fragments davantage. C'est là une présentation en synecdoque, optimiste, positive du problème des fragments: les fragments peuvent constituer un tout et vous ne pouvez briser les fragments. Pour ce qui nous concerne, il s'agit d'une fragmentation initiale: toute cuvre est totalement fragmentée par rapport à cette reine Sprache avec laquelle elle n'a rien en commun et toute traduction est totalement fragmentée par rapport à l'original. La traduction est le fragment d'un fragment, elle brise le fragment - de sorte que le récipient ne cesse d'être brisé - et jamais ne le reconstitue. Il n'y avait au départ pas de vase ou encore: nous n'en avons aucune connaissance, aucune conscience, nous n'y avons pas accès, de sorte que il n'y en eut virtuellement jamais aucun.

C'est pourquoi la distinction entre le symbole et ce qui est symbolisé, la non-adéquation du symbole à un symbolisé en miettes, le caractère non symbolique de cette adéquation, cela est une version des autres distinctions et montre la faiblesse de la rhétorique comme système de tropes qui serait producteur de sens. Le sens est toujours déplacé par rapport au sens qu'il intentionnait idéalement - ce sens $n$ 'est jamais atteint. Benjamin aborde la question dans les termes de l'aporie entre liberté et fidélité, la question qui hante le problème de la traduction. La traduction doit-elle être fidele ou doit-elle être libre? Pour ce qui concerne la pertinence idiomatique de la langue cible, elle doit être libre; par ailleurs, elle doit être relativement fidèle à l'original. La traduction fidèle, qui est toujours littérale, comment peut-elle aussi être libre? Elle ne peut l'être que si elle révèle l'instabilité de l'original et si elle révèle cette instabilité comme la tension linguistique entre le 
trope et le sens. La langue pure est peut-être davantage présente dans la traduction que dans l'original mais sous le mode du trope. Benjamin, qui traite de l'incapacité du trope à être adéquat au sens, utilise constamment les mêmes tropes qui semblent postuler l'adéquation entre le sens et le trope; mais il les en empêche d'une certaine façon, il les déplace de telle sorte que l'original est dynamisé, dé-canonisé, mis en mouvement, un mouvement de désintégration, de fragmentation. Ce mouvement de l'original est un vagabondage, une errance ${ }^{17}$, une sorte d'exil permanent si vous voulez, mais ce n'est pas réellement un exil car il n'y a pas de mère-patrie, quelque chose d'où on a été exile. Il n'y a surtout pas quelque chose comme une reine Sprache, une langue pure, qui n'existe pas sauf en tant que disjonction permanente qui habite tous les langages en tant que teis, y compris et spécialement le langage que l'on considère nôtre. Ce qui apparaît notre langage propre est le plus déplacé, le plus aliéné de tous.

Or c'est ce mouvement, cette errance du langage qui n'atteint jamais le but, qui est toujours déplacé par rapport à ce qu'il est censé atteindre, c'est cette errance du langage, cette illusion d'une vie qui n'est qu'une après-vie, que Benjamin appelle l'histoire. En tant que telle, l'histoire n'est pas humaine car elle appartient strictement à l'ordre du langage; elle n'est pas naturelle pour la même raison; ce n'est pas un phénomène dans la mesure où aucune connaissance, aucun savoir ne peut être retiré d'une histoire qui en tant que telle n'est qu'une complexité linguistique. Elle n'est pas non plus temporelle car la structure qui l'anime n'est pas une structure temporelle. Ces disjonctions du langage sont effectivement exprimées par des métaphores temporelles mais ce ne sont que des métaphores. La dimension future, par exemple, qui est présente, n'est pas temporelle mais dérive de la structure en figures et du pouvoir disjonctif que Benjamin situe dans la nature du langage. L'histoire, telle que Benjamin la conçoit, n'est certainement pas messianique puisqu'elle est formée de la stricte séparation du sacré et du poétique, de la reine Sprache et du langage poétique, et de la réalisation de cette séparation. Reine Sprache, la langue sacrée, n'a rien en commun avec le langage poétique; le langage poétique ne lui ressemble pas, le langage poétique ne dépend pas d'elle,

17. En français dans le texte. 
le langage poétique n'a rien à voir avec elle. C'est cette connaissance négative de son rapport à la langue du sacré qui offre son cadre à la naissance du langage poétique. C'est, si vous voulez, un moment nécessairement nihiliste et qui est nécessaire à toute compréhension de l'histoire.

Benjamin le dit le plus clairement possible non dans cet essai mais dans un autre texte intitulé «Fragment théologico-politique» duquel je citerai un court passage en conclusion. Il le disait le plus clairement possible, me semblait-il, jusqu'à ce que j'essaie de traduire ce passage et $\mathrm{j}$ 'ai trouvé dans l'anglais une particularité qui le rend impossible à traduire. Voici le passage:

Seul le messie lui-même met une fin à l'histoire, en ce sens qu'il libère, qu'il accomplit totalement la relation entre l'histoire et le messianique. C'est pourquoi aucune chose réllement historique ne peut d'elle-même vouloir avoir un rapport avec le messianique. C'est pourquoi le royaume de Dieu n'est pas le telos de la dynamique de l'histoire, il ne peut pas être posé comme son but. Vu historiquement, ce n'est pas son but mais sa fin. ${ }^{18}$

C'est là que j'ai de grandes difficultés avec l'anglais car le mot anglais pour «but» [aim] peut aussi signifier «fin» [end]. Vous dites «la fin et les moyens" [the end and the means], le but et les moyens pour y parvenir. Et le mot anglais «end» peut signifier aussi bien Ziel [but] que Ende [fin]. Ma fin, mon intention. De sorte que si vous voulez utiliser cette tournure idiomatique, la traduction devient: «Vu historiquement, ce n'est pas sa fin mais sa fin» [Seen historically it is not its end but its end], sa terminaison - ce serait du parfait anglais. Mais cela indiquerait que la séparation soulignée par Benjamin est occultée dans le mot «end» [fin] en anglais, qui remplace «but» par «fin», les deux choses que Benjamin nous demande de tenir rigoureusement séparées.

Il ne peut pas être posé comme son but. Vu historiquement, ce n'est pas son but mais sa fin, sa terminaison. C'est pourquoi

18. Nous avons ici traduit en français la traduction en anglais que fait Paul de Man. 
l'ordre du profane ne peut être construit à partir de l'idée du sacré. C'est pourquoi la théocratie n'a pas de sens politique mais uniquement un sens religieux.

Et Benjamin ajoute:

Avoir nié la signification politique de la théocratie, avoir nié la signification politique de la vision religieuse, messianique, avoir nié tout cela avec toute la force nécessaire, c'est le grand mérite du livre de Bloch, l'Esprit de l'utopie.

Puisque nous avons vu que ce qui ici est appelé politique et historique n'est dû qu'à des causes purement linguistiques, nous pouvons remplacer dans ce passage «politique» par «poétique», au sens d'une poétique. Car nous voyons maintenant que le non-messianique, le non-sacré, c'est-à-dire l'aspect politique de l'histoire résulte de la structure poétique du langage, de sorte que politique et poétique sont ici interchangeables, en opposition à la notion de sacré. Dans la mesure où une telle poétique, une telle histoire, n'est pas messianique, non une théocratie mais une rhétorique, elle ne peut accueillir certaines notions historiques telles que celle de modernite, qui est toujours une notion dialectique, c'est-à-dire essentiellement théologique. Vous vous souvenez que nous sommes partis de la proposition de Gadamer sur la modernité, sous la forme d'une dialectique qui était explicitement liée au mot «esprit», à la dimension d'esprit dans le texte de Hegel. Nous avons vu - et il est satisfaisant pour moi d'avoir trouvé - que Hegel lui-même, quand dans l'Esthétique il appuie le sublime sur la même séparation entre sacré et profane, est en fait bien plus proche de Benjamin dans «la Tâche du traducteun qu'il ne l'est de Gadamer. Je terminerai sur cette remarque et je serai heureux de répondre à vos questions si vous le désirez. Merci beaucoup.

Traduit par Alexis Nouss. 